\title{
Sensation magnitude of vibrotactile stimuli ${ }^{1,2}$
}

\author{
RONALD T. VERRILLO ${ }^{3}$ ANTHONY J. FRAIOLI, AND ROBERT L. SMITH \\ SYRACUSE UNIVERSITY
}

\begin{abstract}
A systematic investigation of the subjective magnitude of vibrotaction was undertaken to: (1) determine the growth of sensation as a function of stimulus intensity; (2) establish contours of equal subjective magnitude; and (3) compare over a wide range of frequency and intensity the psychophysical methods of direct scaling and intensity matching. The results show that the data obtained by direct scaling are comparable to the data obtained by interfrequency matching. The subjective magnitude function is a power function with a slope of about 0.89 for frequencies up to $350 \mathrm{~Hz}$. Near threshold the growth of sensation is proportional to the physical intensity. Contours of equal subjective magnitude for vibration across 10 frequencies and at 11 levels of intensity are given.
\end{abstract}

The investigation of the response characteristics of cutaneous mechanoreception at suprathreshold levels has been largely motivated by efforts to understand auditory phenomena better (Békésy, 1960) and by attempts to develop cutaneous communication systems (Geldard, 1961). There have been few attempts to investigate systematically the skin as a surface, sensitive to mechanical disturbances, that functions primarily at suprathreshold levels of stimulation.

The experiments described in this report aimed at a systematic determination of some psychophysical vibrotactile characteristics at suprathreshold levels. More specifically they include: (1) A determination of functions of subjective intensity by means of the method of psychological magnitude balance (Hellman \& Zwislocki, 1963), which is based on the scaling methods of S.S. Stevens (Stevens, 1955, 1956). (2) A determination of frequency contours of equal subjective magnitude. The contours are analogous to the well known equal-loudness contours determined by Fletcher and Munson (1933) and others.

Although loudness-level functions have been extensively investigated in hearing, there have been few attempts to establish similar functions for taction. Goff (1967) has obtained two contours of equal subjective intensity in her study on vibrotactile frequency discrimination. Bekésy (1955) has shown that, at suprathreshold levels, the subjective intensity for cutaneous vibration increases more rapidly over areas of low neural density than over areas of high neural density, a phenomenon that may be considered as analogous to loudness recruitment in hearing. Stevens (1968), with a matching procedure, was able to construct a series of equal sensation contours for vibration covering a range of five frequencies between 20 and $320 \mathrm{~Hz}$ and at seven levels of intensity. This is the most complete set of contours available to date.

While all of these studies indicate that it is possible to establish for vibrotaction a set of equal sensation contours comparable to those available for hearing, they do not provide a sufficient range of frequencies and intensities systematically investigated under rigorously controlled experimental conditions.

The direct scaling of tactile magnitudes has received considerably more attention. S. S. Stevens (1959) reported a series of experiments from which functions have been generated that relate subjective responses to stimulus intensities. Using the method of magnitude estimation with a designated standard, he demonstrated that his power law (Stevens, 1957) adequately describes the relationship between subjective intensity and the amplitude of vibration at the fingertip. Using the same psychophysical method, Sherrick (1960) and Franzén (1966) obtained results that agreed with those of Stevens.

The method of magnitude estimation with a designated standard tends to produce biased results. Stevens (1956) reported that, in auditory experiments, Ss appear to favor moderate intensity levels and tend to select numbers that center about the designated standard. Reynolds and Stevens (1960) showed that this method produces a function with a smaller exponent than that produced by the method of magnitude production. Hellman and Zwislocki (1961) demonstrated that the relationship between the designated reference number and a reference sensation level can have a pronounced effect upon the shape of the resulting loudness function. The relationship is such that when the reference number is subjectively too large for a given reference sensation level, a flattened loudness curve is produced above the standard and a steeper one below. The reverse is true when the reference number is too small. As a result of such biases, Stevens (1956) and Hellman and Zwislocki (1963) suggest that the reference standard be omitted in the determination of subjective sensory magnitudes.

An important detail in the determination of subjective magnitude functions is the relationship between sensory magnitude and intensity near threshold. It is not possible to determine from the published data of Stevens (1957), Sherrick (1960), or Franzén (1966) how near to the threshold of detectability they carried their scaling experiments. Magnitude estimates made near the threshold are important for several reasons. In hearing, it has been shown that the loudness function near threshold becomes steeper than at higher intensity levels (Stevens, 1955; Hellman \& Zwislocki, 1961; Scharf \& Stevens, 1961; and others). This steepening affects the analytical expression for the loudness function (Ekman, 1956; Zwislocki, 1965; Marks \& J. C. Stevens, 1968). Zwislocki's (1960) theory of temporal summation requires that, near threshold, the sensory magnitude be approximately proportional to the stimulus intensity. The mathematical rationale for this requirement is given in detail by Zwislocki (1965) with supporting experimental documentation. The interaction of signal and noise at low stimulus intensities is sufficient to explain the steepening of the loudness function near threshold.

\section{INSTRUMENTATION AND METHOD}

Vibrotactile stimulation was provided by a Goodmans $360 \mathrm{~A}$ vibrator. The $S$ and the vibrator were located inside a booth to minimize unwanted environmental noise and vibrations. The vibrator was located on the platen of a drill press assembly that permits fine adjustments in the position of the vibrator in relation to the S's hand. The contactor, mounted on the moving element of the vibrator, protruded through a hole in a rigid surface positioned over the vibrator. There was a $1-\mathrm{mm}$ gap between the contactor and the rigid surface.

The $S$ sat next to the vibrator with his right forearm resting comfortably on the rigid surface at right angles to the direction of vibration. His hand was positioned over the hole and contactor. The amplitude of vibration was monitored by means of a carefully calibrated accelerometer mounted directly on the moving element of the vibrator. Acceleration voltages were converted to displacement in microns by a double integration and converted to decibels. (See Verrillo, 1963, 1966, and 1968, for 
more detailed descriptions of the apparatus.)

The following apparatus was used in the determination of numerical magnitude balance functions. The output of a sine-wave generator was modulated by an electronic switch. The $O N$ and OFF sequence of the switch was determined by two waveform generators and two pulse generators. The output of the switch was preamplified and fed to an attenuator under the control of the E. The signal then passed through a 120-dB continuously variable attenuator, located inside the booth, by which the $S$ could alter the intensity of vibration. After a final stage of amplification, the signal activated the vibrator.

The stimuli were sinusoidal bursts of $600-\mathrm{msec}$ duration, with a rise-fall time of about $10 \mathrm{msec}$ and a silent interval of $1,400 \mathrm{msec}$ between bursts. The vibrations were presented to the thenar eminence of the right hand via a $2.9 \mathrm{~cm}^{2}$ contactor mounted on the vibrator. The $\mathrm{Ss}$ wore earphones delivering a narrow-band random noise to mask the sound of the vibrator.

\section{Scaling Subjective Intensity}

Subjective intensity functions were determined by the direct judgment of stimulus magnitudes. The method of numerical magnitude balance was used (Hellman \& Zwislocki, 1963), which is the nonnormalized combination of the methods of magnitude estimation and magnitude production (Stevens, 1955, 1958). Six Ss were first required to assign numbers (magnitude estimation) to a randomly presented series of 10 stimulus intensities. Stimulus intensities ranged from 2 to $50 \mathrm{~dB}$ above the measured threshold at any given frequency. Subjective intensity functions for 10 frequencies were determined $(25,40,64,100,150,200$, $250,350,500$, and $700 \mathrm{~Hz}$ ). No reference standard was used in order to minimize the biases discussed above. The Ss were instructed "to assign numbers which appear to be representative of the subjective intensity, or the strength of the vibration." A random series of intensities was presented three times at each frequency and the first run of each set was discarded. The geometric mean of Runs 2 and 3 was taken as the assigned number. The data were then plotted in terms of assigned number as a function of sensation level.

Following the completion of this task by all Ss, the psychological magnitude of vibration was matched (magnitude production) to numbers presented in random order by the $\mathbf{E}$. The numbers presented were obtained by taking the geometric mean of the numbers assigned by all $\mathrm{Ss}$ in magnitude estimation. The $\mathrm{S}$ controlled the amplitude of vibration by means of a plain knob connected by a shaft to a $120-\mathrm{dB}$ continuously variable attenuator located outside the booth. After receiving instructions on the use of the control, the $S$ was instructed "to adjust the signal so that its magnitude appears to subjectively fit the number called." At every testing session, three runs of 10 randomized numbers were presented. The first run was discarded and the geometric means of the attenuator settings of Runs 2 and 3 were taken as the produced intensities.

The results of magnitude estimation and magnitude production were averaged (geometric mean) from group data to produce the magnitude balance function. Contours of equal subjective intensity were derived by replotting the data in terms of displacement as a function of frequency.

\section{Matching By Adjustment}

Contours of equal subjective intensity were obtained by a direct matching procedure using the method of adjustment. Prior to each experimental session, the absolute threshold was determined for each of the two frequencies to be matched. The intensity of a standard frequency was set at 1 of 11 predetermined intensity levels above the S's threshold. The $S$ was asked to match, by adjusting a variable attenuator, the subjective intensity of the test frequency to that of the standard.
The standard and test frequencies were presented alternately until the $S$ indicated that a match had been made. Time values of the stimulus were identical to those used for subjective intensity scaling. Following the completion of each match, the intensity of the standard frequency was changed in a random sequence and the procedure was repeated until all the experimental intensity levels above threshold were tested. The 11 intensities were presented three times and the first run was discarded. The geometric mean setting of Runs 2 and 3 constituted the intensity match. In order to compensate for a possible bias, the entire procedure was reversed, and the intensity of the standard frequency was varied by the $\mathrm{S}$. Two standard frequencies ( 64 and $250 \mathrm{~Hz}$ ) were used in order to reduce the biases that may result from the comparison of widely disparate frequencies (Ross, 1965). Contours of equal sensation magnitude were plotted as vibration amplitude as a function of frequency for each of the two standard frequencies and, finally, the two families of curves were combined to a single set of contours.

The instrumentation for the matching procedures was identical to that used for scaling except for the addition of a second channel and a mixer. The Ss controlled the onset and termination of the train of stimuli with a foot switch.

\section{Order Effect}

Prior to the systematic investigation of sensation magnitudes, it was decided to determine the effect of stimulus order upon subjective judgments of magnitudes. The "time error" or order effect is well known and often mentioned in textbooks of experimental psychology. In its simplest form, the effect (it is not really an "error" as the older term implies) stems from the influence of one stimulus upon another in a paired situation. There is a systematic under- or overestimation of one of the pair depending on whether the magnitude of the first or the second (among other factors) is being estimated or detected.

It became imperative to determine the extent of the order effect under conditions used in the direct intensity matching described above. Pairs of sinusoidal vibration bursts were presented to the thenar eminence of the right hand with the same experimental setup used in the other experiments. The burst duration of each unit was $600 \mathrm{msec}$ with a time interval of 1,000 msec between bursts of a single pair. A time interval of $1,800 \mathrm{msec}$ separated pairs of stimuli. A frequency of $250 \mathrm{~Hz}$ was used for both units in each pair. Two separate signal channels were fed to the vibrator through a mixer. The signal intensity in one of the channels was under the direct control of the $S$ through a 120-dB continuously variable attenuator.

The $S$ was asked to adjust the subjective intensity of one signal in each pair to the subjective intensity of the other, which was set by the investigator. Standard intensities of $6,10,20,30,40$, and $50 \mathrm{~dB}$ sensation level were presented in random order. In two separate sessions the $S s$ matched intensities of the first to the second and of the second to the first unit of each pair. The order of matching was balanced between the six Ss used.

\section{Test of Procedure}

The procedures for the scaling of vibratory intensity were patterned after those developed for the scaling of loudness in hearing (Stevens, 1956; Robinson, 1957; Scharf \& Stevens, 1961; Hellman \& Zwislocki, 1963). In order to test the validity of the procedure, seven $S s$ judged the subjective magnitude (magnitude estimation) of a $1000-\mathrm{Hz}$ tone presented monaurally. The resultant function was compared to data reported by a number of other investigators.

\section{RESULTS AND DISCUSSION}

The results of the experiments on the order effect and cross-modality matching are presented first since their purpose 


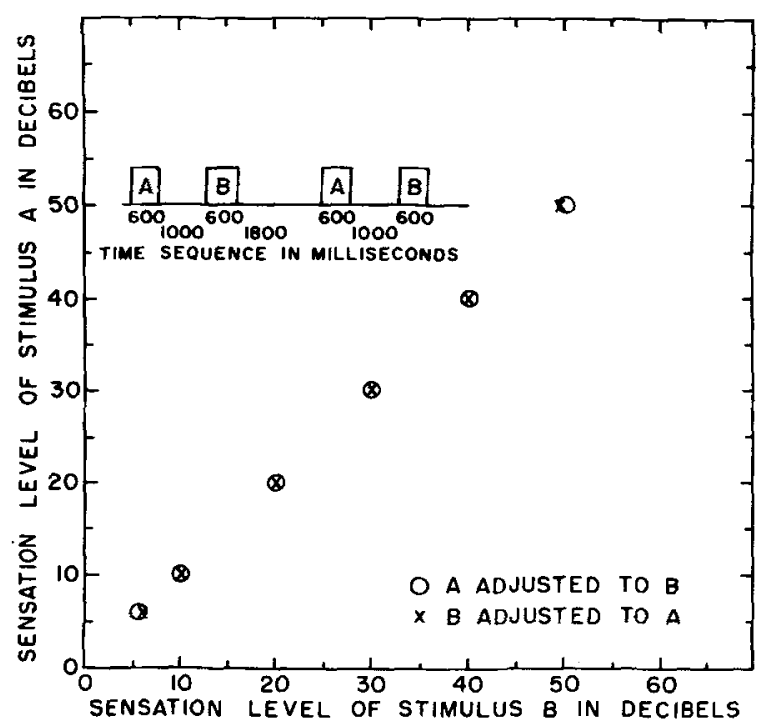

Fig. 1. Effect of the order of presentation of the standard stimulus upon the intensity matching of the variable X-A as standard; $O-B$ as standard; $f=250 \mathrm{~Hz}$.

was to clarify several methodological questions prior to performing the main experiments.

\section{Order Effect}

Two curves were obtained: one resulting from matching the first burst to the second and one resulting from matching the second burst to the first. The results are plotted in terms of the sensation level of the first burst vs the sensation level of the second burst (Fig. 1). The agreement between matching sequences is almost perfect. There does not appear to be any effect of order upon perceived magnitude under these conditions. It is assumed, then, that the order effect would be absent also in the experiments where stimuli are presented with equal temporal spacing.

\section{Test of Procedure}

Magnitude estimation of a $1000-\mathrm{Hz}$ tone presented to the right ear results in a power function with a slope of 0.54 . This is in excellent agreement, both in slope and in position, with the data summarized by Hellman and Zwislocki (1963). Their summary included a total of 15 studies done at a number of different laboratories. The close agreement with previous investigations was accepted as a validation of the testing procedures used in the present experiments.

\section{Scales of Subjective Intensity}

The raw data for subjective magnitude in assigned numbers were plotted directly as a function of sensation level in decibels for both magnitude estimation and magnitude production. For every frequency tested, the curve drawn through the geometric means of the individual responses was a power function. When the functions for magnitude estimation and magnitude production for each frequency were plotted together and averaged, curves of numerical magnitude balance were obtained. The resulting curves were all power functions with exponents ranging from 0.89 to 1.2 . The value of the exponent for frequencies between $25 \mathrm{~Hz}$ and $350 \mathrm{~Hz}$ inclusive was approximately 0.89 ; at $500 \mathrm{~Hz}$ it was 0.95 , and at $700 \mathrm{~Hz}$ the exponent was 1.2.

Figures 2 and 3 show the raw data for magnitude estimation and magnitude production, respectively, for a single frequency

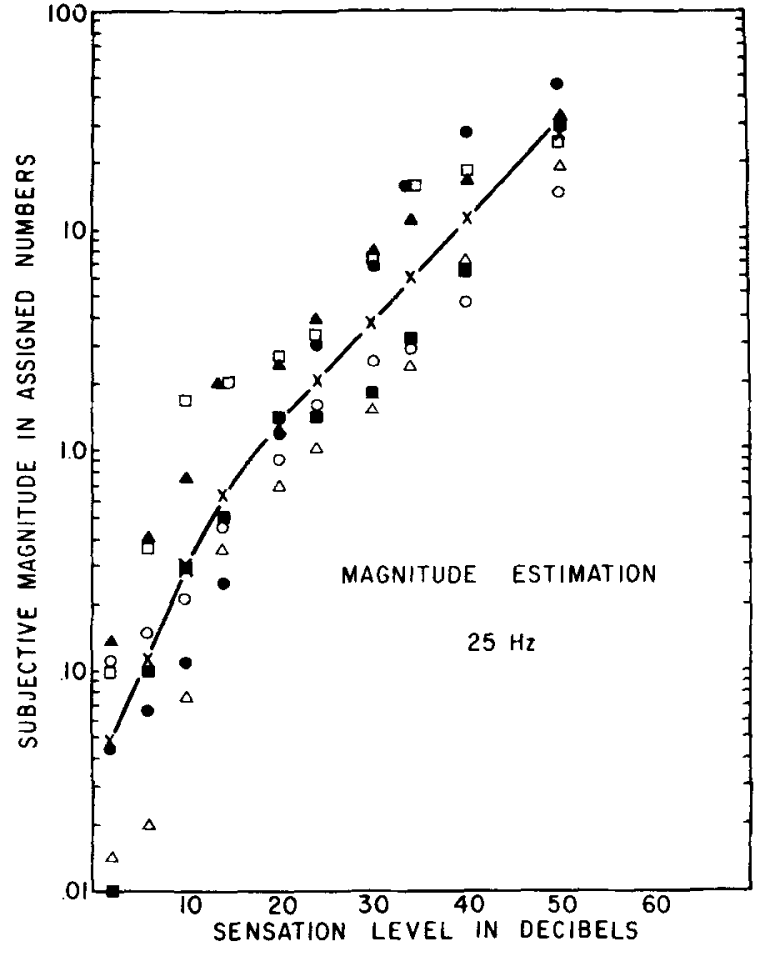

Fig. 2. Numerical magnitude estimation of subjective vibration intensity as a function of sensation level. Symbols represent individual raw data. A solid line has been drawn through the geometric means $(\mathbf{X})$.

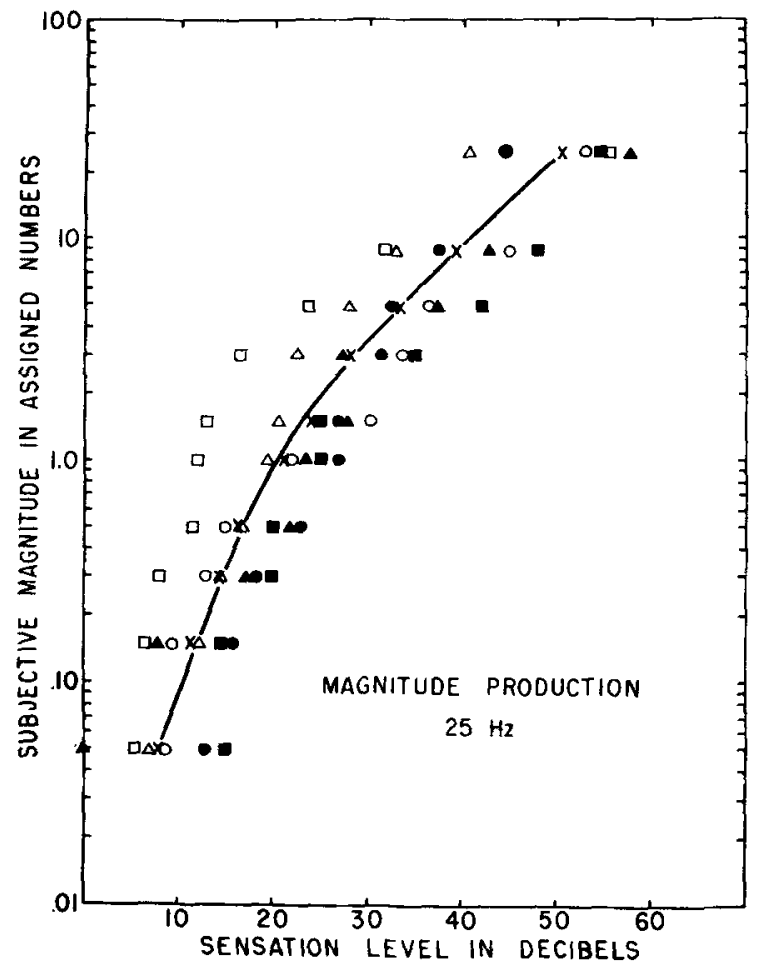

Fig. 3. Subjective magnitude production for assigned numbers. Symbols represent individual raw data. A solid line has been drawn through the geometric means (X). 
$(25 \mathrm{~Hz})$. Curves of best fit have been drawn through the geometric means. Each symbol represents the responses of a single $\mathrm{S}$.

Figures 4 and 5 illustrate the geometric means for magnitude estimation and magnitude production for $40 \mathrm{~Hz}$ and $250 \mathrm{~Hz}$, respectively. A solid line representing the magnitude balance function has been drawn for each frequency. These two frequencies were selected for illustration because they represent the extremes of coincidence obtained between magnitude estimation and production curves.

A measure of the extent of variability among individual responses across all frequencies is shown in Fig. 6 . The curve describes the average mean values of assigned numbers for frequencies between 25 and $350 \mathrm{~Hz}$. This averaging seems permissable since the value of the exponent for all frequencies included was the same $(0.89)$. The solid and dashed bars illustrate the average deviations from the mean for magnitude estimation and magnitude production, respectively. There is an inverse relationship in the size of the deviations for estimation and production as a function of sensation level. At low levels of sensation, the estimation variability is large and the production variability is relatively small. Estimation variability decreases while production variability increases across sensation levels. A rectangle can be described whose sides are parallel to the coordinate axes and bounded by the average deviations at each sensation level. The slope of the diagonal of each rectangle will closely approximate the slope of the magnitude balance function in that region. This may be interpreted as evidence that the variance for both estimation and production is attributable to a single source, that is, the individual's judgment of magnitude regardless of the task performed in making that judgment.

The magnitude balance curves of four frequencies are shown in Fig. 7 as a function of absolute displacement rather than sensation level to show the relative placement of the curves on an absolute scale. Several significant features of the results are illustrated in these curves. First, the slopes near threshold are approximately parallel for all frequencies. (This is true of all the frequencies tested, including those not shown in Fig. 7.) Furthermore, there is a direct proportionality between the subjective magnitude and the intensity of the stimulus. For every doubling of the stimulus intensity, the perceived intensity of the stimulus increases twofold. This confirms directly a requirement of Zwislocki's (1960) theory of temporal summation and its extension to include the summation of loudness (Zwislocki, 1965). The theory states that the sensory magnitude is approximately proportional to the stimulus intensity near threshold.

Another feature of the curves shown in Fig. 7 is the longer steep segment of the curve for $25 \mathrm{~Hz}$. This is a distinctive feature of the low frequencies and may reflect the activity of a different type of receptor. There is considerable evidence supporting a duplex system of receptors in cutaneous tissue subserving mechanoreception (Verrillo, 1965, 1968; Zwislocki, 1968). The extended steep portion of the subjective magnitude function may be a further manifestation of the dynamic characteristic of a low-frequency receptor system distinct from the system operating at higher frequencies. Further research is needed to clarify this finding.

Finally, the curves in Fig. 7 show parallel functions at the upper intensities of each frequency except $500 \mathrm{~Hz}$, which is steeper (0.95). The slope for $700 \mathrm{~Hz}$ (not shown) is also steeper (1.2). The independence of slope and frequency does not agree with results reported by other laboratories. Stevens (1959) found that slope varied with frequency when he tested at 60 and $120 \mathrm{~Hz}$ and obtained slopes of 0.83 and 0.94 , respectively. More recently, Stevens (1968), using a matching procedure, again reported a decrease of exponent for frequencies up to $250 \mathrm{~Hz}$.

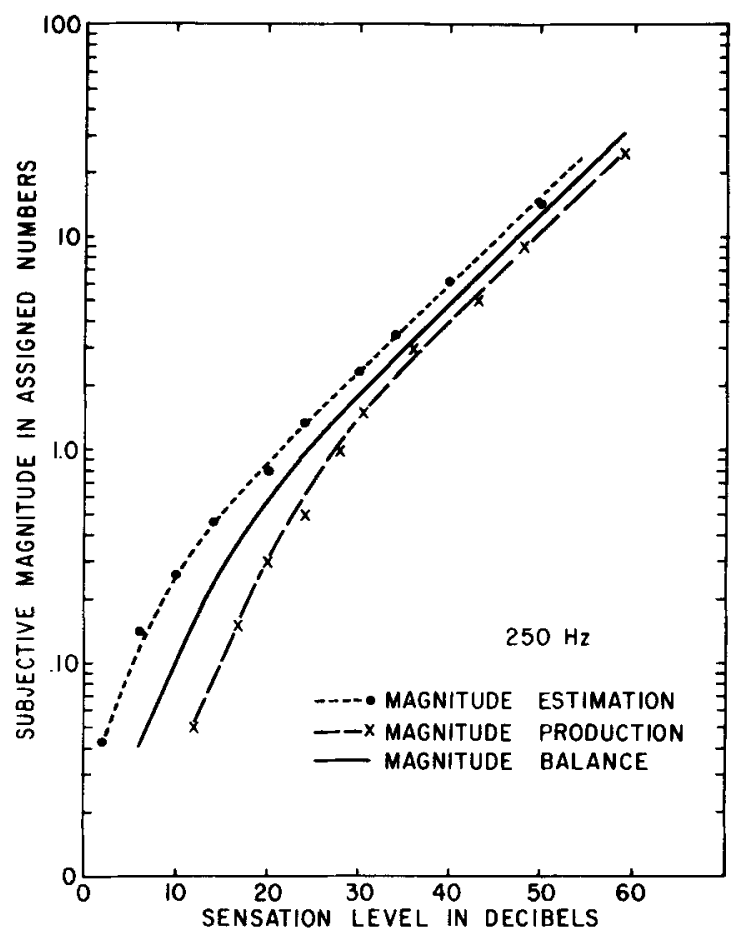

Fig. 4. Geometric means for magnitude estimation ( $\bullet$ ) and magnitude production (x) at $40 \mathrm{~Hz}$. The solid line represents the numerical magnitude balance curve.

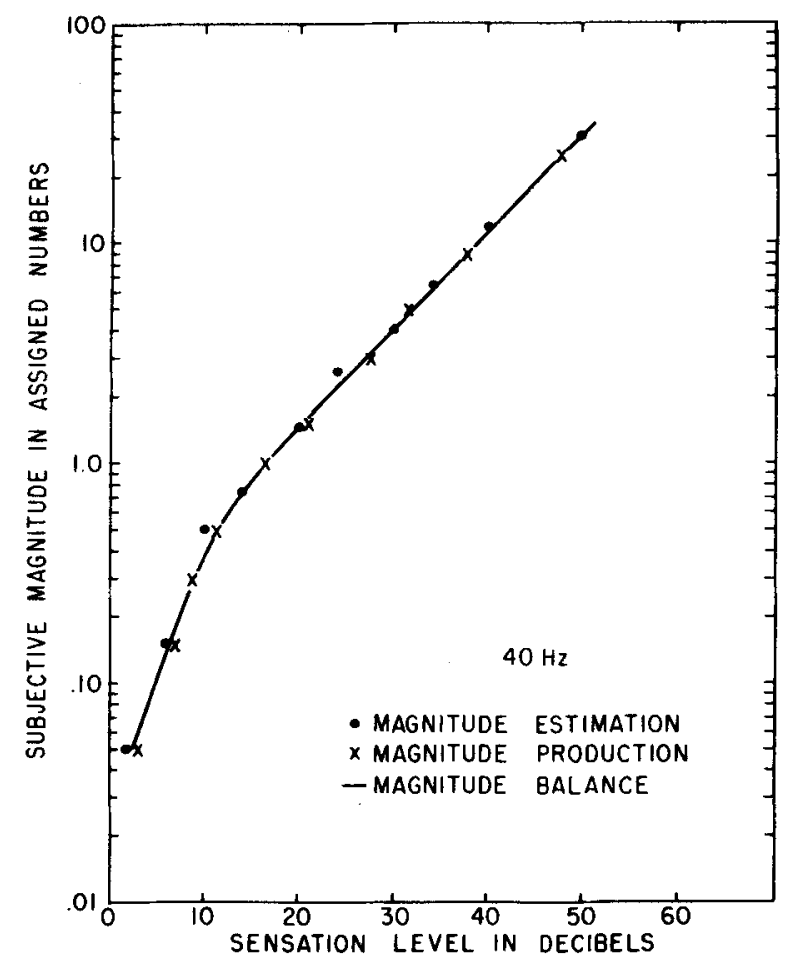

Fig. 5. Geometric means for magnitude estimation ( () and magnitude production (x) at $250 \mathrm{~Hz}$. The solid line represents the numerical magnitude balance curve bisecting the curves for estimation and production. 


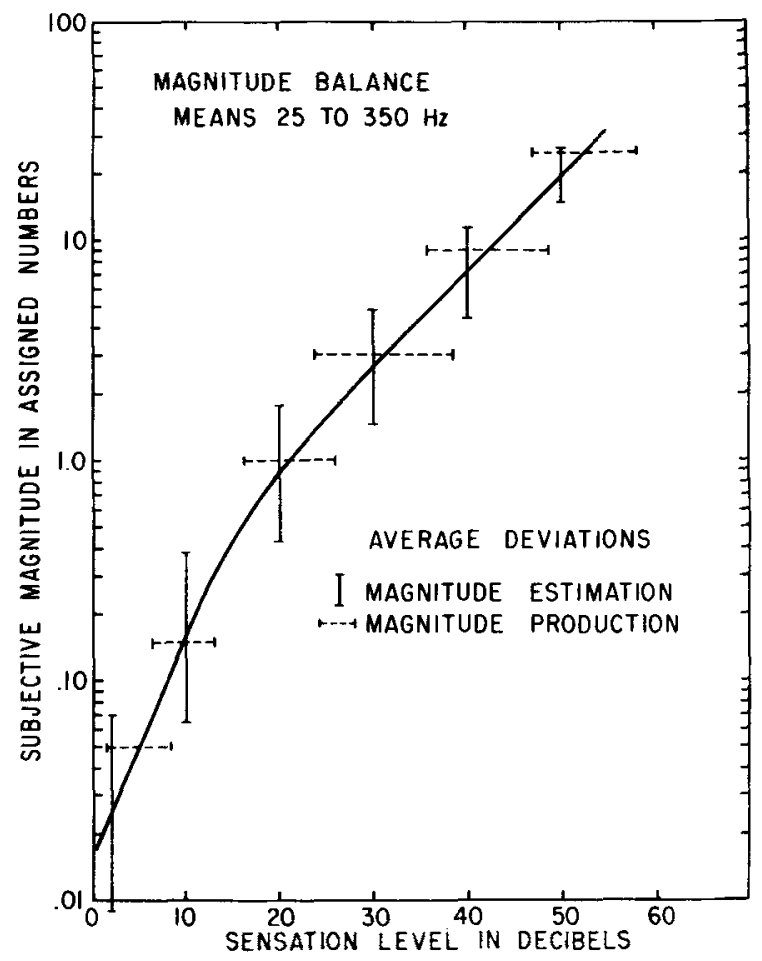

Fig. 6. Average magnitude balance curve for frequencies 25 to $350 \mathrm{~Hz}$ inclusive. Average deviations at six sensation levels are shown for magnitude estimation (solid vertical bars) and magnitude production (dashed horizontal bars).

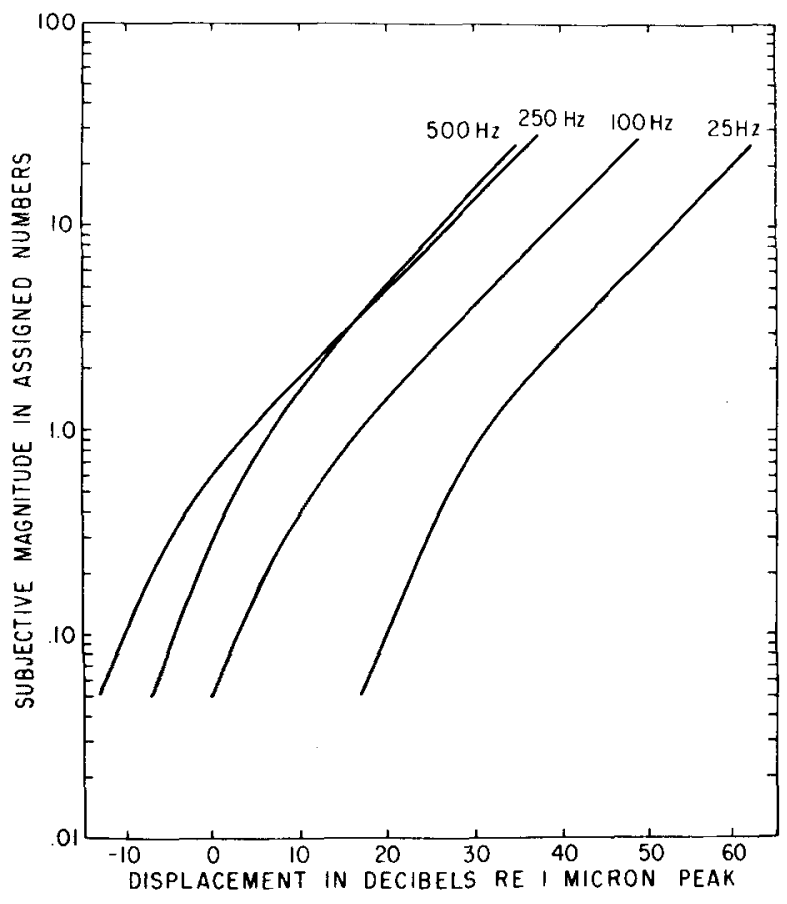

Fig. 7. Magnitude balance curves for four frequencies plotted as a function of absolute displacement.

It is not possible at this time to reconcile the differences obtained by the different laboratories. The most obvious explanation is one of procedure. The measurements of Stevens were obtained on the pad of the finger tip, using a standard intensity with an assigned numerical value and having no limiting surround. Furthermore, experiments using suprathreshold levels of stimulus amplitude have been limited by the design characteristics of mechanical vibrators. This limitation takes the form of a dc thrust or pedestal at the start of the burst of a sinusoidal signal and "ringing" at the offet of the signal. These distortions of the signal became particularly evident and, in all likelihood, significant at high intensities for frequencies above about $300 \mathrm{~Hz}$. Until the mechanical problems are remedied and experiments on the finger tip (and other body sites) are performed under more precisely controlled conditions, there will be no adequate explanation for the disparate results.

\section{Contours of Equal Sensation Magnitude}

All of the data collected in the direct scaling experiments were transformed into absolute displacement values and replotted in terms of displacement as a function of frequency. The resulting family of curves (Fig. 8) illustrates the stimulus intensity required to obtain a constant sensation magnitude as a function of frequency. The derived curves are analogous to the well-known equal loudness contours in audition.

Using the procedure described above (matching by adjustment), a set of equal sensation contours was obtained by direct intensity matching among frequencies. The results are shown in Fig. 9. These contours can be compared to corresponding curves derived from the scaling of vibrotactile intensities by the method of psychological magnitude balance. Figure 10 compares the results obtained by the two methods at two suprathreshold intensities. Thresholds were obtained at the beginning of each session. The comparison projects a clear picture of agreement between the two methods. The agreement is interpreted as a clear validation of magnitude balance as a method for determining the dynamic characteristics of vibrotactile stimulation.

An outstanding feature of the contours shown in Figs. 8 and 9 is their similarity in shape to curves obtained for audition. At threshold, the curve is U-shaped with a flattened portion in the frequency range below $40 \mathrm{~Hz}$. There is a gradual departure from this shape with increasing intensity until at the highest intensities the contours assume a smoother, more uniform slope over the entire range of frequencies. The smoothing at high intensities is characteristic in similar contours obtained for audition.

The upper portion of Fig. 11 shows the displacement necessary, at six frequencies, to match subjectively the magnitude of the $250-\mathrm{Hz}$ standard. The lower plot in this figure shows the resulting slope values for all of the frequencies tested. They are all on the order of 1.0 except for the two highest frequencies. The departure may be a function of the sensory characteristic, or an artifact resulting from the inadequate stimulus control discussed above. The slope of about 1.0 for frequencies below $500 \mathrm{~Hz}$ implies that the slopes of the power functions obtained by the method of magnitude balance are independent of frequency. The results have shown that this is indeed the case except for 500 and $700 \mathrm{~Hz}$.

Support for a duplex system of receptors can be derived from the curves for 25 and $40 \mathrm{~Hz}$. Although the threshold at these two frequencies is the same, the sensation magnitude at $40 \mathrm{~Hz}$ grows at a more rapid rate than that at $25 \mathrm{~Hz}$ before leveling off. Less displacement at $40 \mathrm{~Hz}$ is required to equal the sensory magnitude of a $25-\mathrm{Hz}$ signal at suprathreshold levels. The picture is consistent with the hypothesized duplex system, one part of which functions at low frequencies of vibration that can be superseded, as the frequency or intensity of vibration is increased, by a second part that functions at higher frequencies. The relative shapes of the curves for 25 and $40 \mathrm{~Hz}$ in Fig. 11 a suggests an overlap of influence between the two systems in determining the response in the region of $40 \mathrm{~Hz}$. 


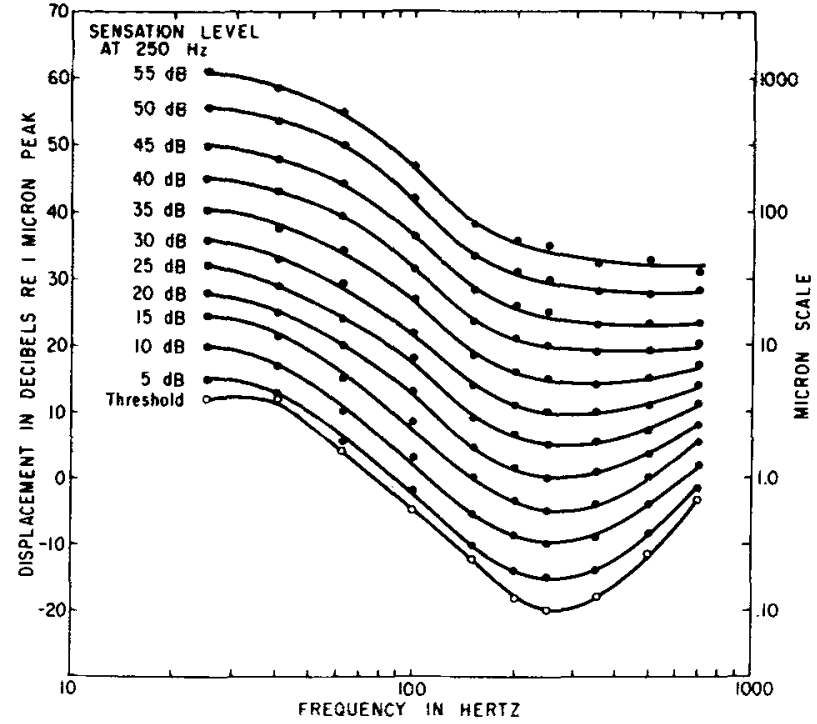

Fig. 8. Contours of equal sensation magnitude for vibration derived from data obtained by the method of numerical magnitude balance. The sensation-level indications refer to $250 \mathrm{~Hz}$.

\section{SUMMARY}

A systematic investigation of the subjective magnitude of vibrotaction was undertaken in order to (1) determine the growth of sensation magnitude as a function of stimulus intensity; (2) establish the contours of equal subjective magnitude that would be comparable to those for hearing; and (3) compare over a wide range of frequencies and intensities the psychophysical methods of direct scaling and intensity matching.

The site of stimulation for all experiments was the thenar eminence of the right hand. Stimulation was provided by a round contactor with an area $2.9 \mathrm{~cm}^{2}$ mounted upon a vibrator. The contactor had a rigid surround that minimized the spread of surface waves over the skin.

Several methodological problems had to be resolved before undertaking the main experiments. The first involved the effect of the order of stimulus presentation in an intensity matching procedure. It was clearly established that, under the experimental

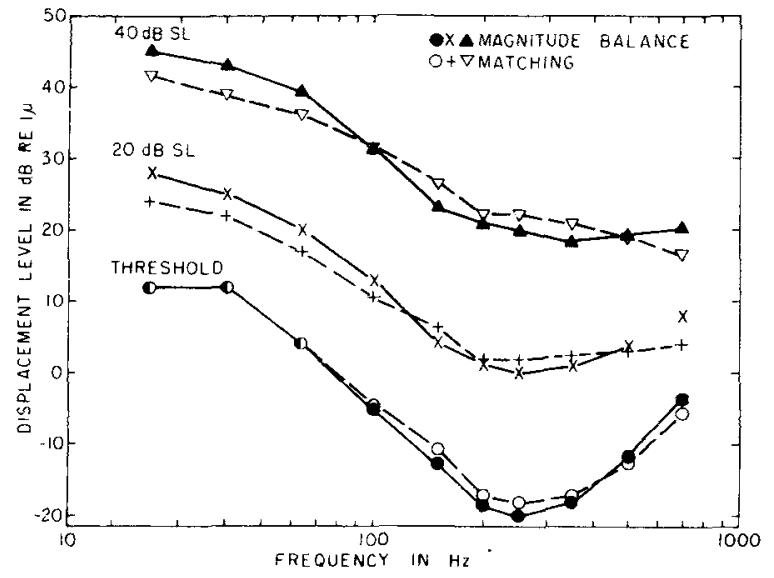

Fig. 10. Comparison of contours of equal sensation magnitude for three levels of sensation obtained by the methods of numerical magnitude balance and direct intensity matching. The reference level indications refer to $250 \mathrm{~Hz}$.

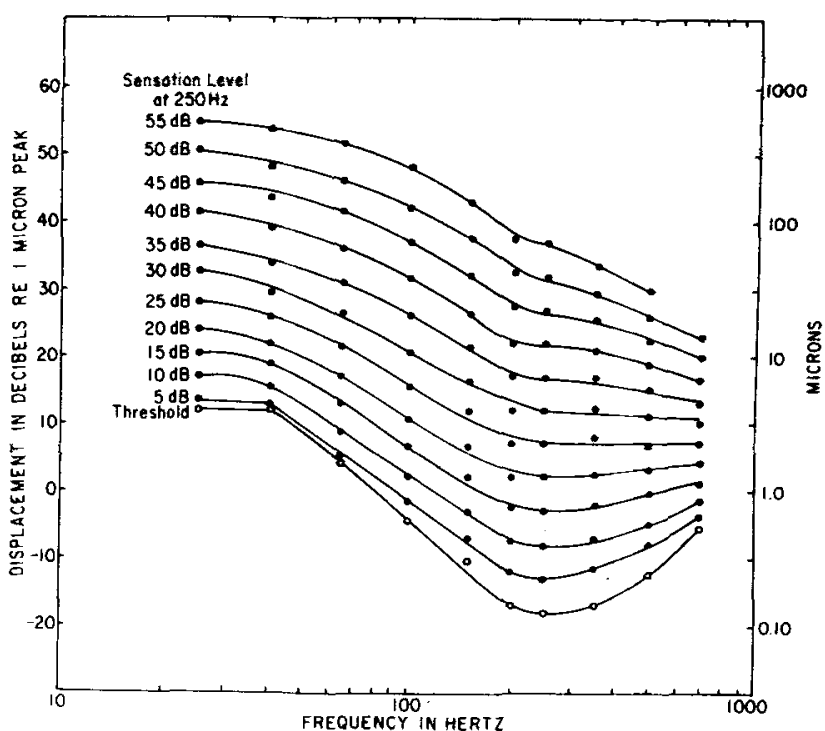

Fig. 9. Contours of equal sensation magnitude for vibration from data obtained by the method of direct intensity matching. The sensation-level indications refer to $250 \mathrm{~Hz}$. Reference frequencies for matching were 64 and $250 \mathrm{~Hz}$.

conditions used, the order effect did not appear. The second problem was to establish the validity of the direct scaling method used in the scaling of sensory magnitudes. The method of numerical magnitude balance was used to determine loudness functions for hearing. The results compared extremely well with similar functions reported out of a number of laboratories and widely accepted by the research community. The close agreement with previous studies was accepted as a verification of the procedures used.
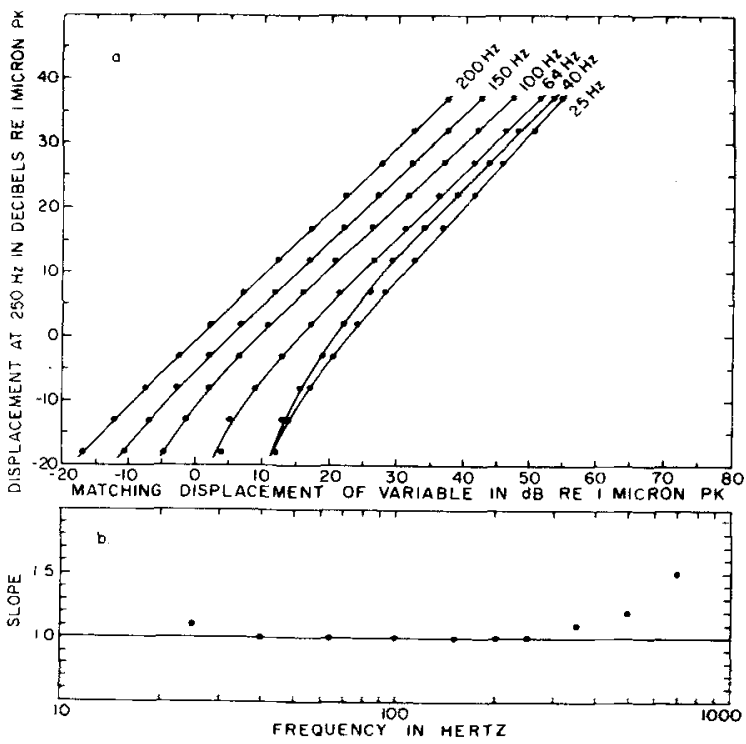

Fig. 11. (a) Regression curves showing displacement of the standard frequency as a function of the displacement required to produce a sensation of equal magnitude for six adjusted frequencies. (b) Slopes of the regression curves (a) for all of the frequencies tested. A slope approximating 1.0 indicates that the slopes of the power functions obtained by the method of magnitude balance are independent of frequency. 
Two psychophysical methods were employed to determine the contours of equal subjective magnitude:

(1) Direct scaling by the method of numerical magnitude balance. Six Ss assigned numbers to a randomly presented series of vibrotactile intensities (magnitude estimation). They were then asked to adjust the intensity of the stimulus to correspond subjectively to a randomly presented series of numbers given by the $\mathrm{E}$ (magnitude production). The resultant functions were averaged, yielding a nonnormalized subjective magnitude function (numerical magnitude balance).

(2) Intensity matching of two different frequencies of vibration by the method of adjustment. In a counterbalanced design, 10 frequencies were matched by six $S$ s at 11 levels of intensity. Two standard frequencies were used in order to avoid possible biases introduced by a high or a low standard frequency.

For the data obtained by direct scaling, the subjective magnitude in numbers at each frequency is plotted as a function of sensation level. The resultant curves are power functions with slopes of approximately 0.89 at frequencies up to $350 \mathrm{~Hz}$. The slopes at $500 \mathrm{~Hz}$ and $700 \mathrm{~Hz}$ increase to 0.95 and 1.2 , respectively. A possible reason for the increase at high frequencies is discussed.

Sensation levels were converted to absolute displacements in decibels and replotted as a function of frequency. The resultant family of curves reflects the equal sensation magnitude at each frequency for 11 different levels of intensity above the threshold at $250 \mathrm{~Hz}$. Contours of equal subjective magnitude were also generated from the data obtained by direct matching. Comparison of the two sets of curves provides an evaluation of direct scaling as a method for determining contours of equal sensory magnitude. The contours obtained by matching and by scaling agree in both shape and intensity level except at high intensities (above $40 \mathrm{~dB}-\mathrm{SL}$ ) in the high-frequency region (500 and $700 \mathrm{~Hz}$ ). The reason for the increased difference is not clear at the present time.

From the contours of equal subjective magnitude of vibration obtained by matching, it is possible to construct a curve at each frequency that plots the displacement required to achieve a given subjective magnitude vs the displacement required for the same subjective magnitude at $250 \mathrm{~Hz}$. For all frequencies below $500 \mathrm{~Hz}$, the slopes of the resultant curves are approximately equal to 1.0. This implies that the slopes of the power functions obtained by the direct scaling method are independent of frequency. This is indeed the case except for 500 and $700 \mathrm{~Hz}$. The curves also support the hypothesis of a duplex mechanism mediating the perception of vibration.

Some general conclusions may be drawn from these studies:

(1) The data obtained by magnitude balance are comparable to the data obtained by interfrequency matching.

(2) The subjective magnitude function for vibrotaction is a power function (Stevens's power law) with a slope of about 0.89 for frequencies up to $350 \mathrm{~Hz}$. Above this frequency, the slope may be increasing with frequency.

(3) Near the threshold of detectability the growth of sensation is proportional to the physical intensity. This is direct confirmation of the prediction made by Zwislocki's (1965) mathematical theory.

(4) Contours of equal subjective magnitude for vibration across 10 frequencies and at 11 levels of intensity have been determined. The contours are analogous to the equal loudness contours in hearing.

\section{REFERENCES}

BÉKESY, G. von. Experiments in hearing. New York: McGraw-Hill, 1960. EKMAN, G. Subjective power functions and the method of fractionation. Report from the Psychological Laboratory, University of Stockholm, 1956, No. 34.

FLETCHER, H., \& MUNSON, W. A. Loudness, its definition, measurement and calculation. Journal of the Acoustical Society of America, 1933, 5 , 82-108.

FRANZÉN, O. On summation: A psychophysical study of the tactual sense. In "Quarterly Progress and Status Report," Speech Transmission Laboratory, Royal Institute of Technology. Stockholm, Sweden, January $15,1966$.

GELDARD, F. A. Cutaneous channels of communication. In W. A. Rosenblith (Ed.), Sensory communication. New York: Wiley, 1961 Pp. 73-87.

GOFF, G. D. Differential discrimination of frequency of cutaneous mechanical vibration. Journal of Experimental Psychology, 1967, 74, $294-299$.

HELLMAN, R. P., \& ZWISLOCKI, J. Some factors affecting the estimation of loudness. Joumal of the Acoustical Society of America, 1961,33, 687-694.

HELLMAN, R. P., \& ZWISLOCKI, J. Monaural loudness function at 1,000 cps and interaural summation. Journal of the Acoustical Society of America, 1963, 35, 856-865.

MARKS, L. E., \& STEVENS, J. C. The form of the psychophysical function near threshold. Perception \& Psychophysics, 1968, 4, 315-318.

MOUNTCASTLE, V. B., TALBOT, W. H., DARIAN-SMITH, I., \& KORNHUBER, H. H. Neural basis of the sense of flutter-vibration. Science, 1967, 155, 597-600.

REYNOLDS, G. S., \& STEVENS, S. S. Binaural summation of loudness. Journal of the Acoustical Society of America, 1960, 32, 1337-1344.

ROBINSON, D. W. The subjective loudness scale. Acustica, 1957, 7, 217.

ROSS, S. Loudness of pure tones as a function of frequency, intensity, and middle ear mechanics. Part I. Matching functions for loudness. Center for Research on Language and Language Behavior. University of Michigan, Ann Arbor, Mich, 1965.

SCHARF, B., \& STEVENS, S. S. The form of the loudness function near threshold. In L. Cremer (Ed.), Proceedings of the Third International Congress on Acoustics, 1959. Amsterdam: Elsevier, 1961.

SHERRICK, C. E. Observations relating to some common psychophysical functions as applied to skin. In G. R. Hawkes (Ed.), Symposium on cutaneous sensitivity. Ft. Knox, Ky.: U.S. Army Medical Research Laboratory, 1960. Pp. 147-158.

STEVENS, S. S. The measurement of loudness. Journal of the Acoustical Society of America, 1955, 27, 815-829.

STEVENS, S. S. The direct estimation of sensory magnitude loudness. American Journal of Psychology, 1956, 69, 1-25.

STEVENS, S. S. On the psychphysical law. Psychological Review, 1957, 64, 153-181.

STEVENS, S. S. Problems and methods of psychophysics. Psychological Bulletin, 1958, 54, 177-196.

STEVENS, S. S. Tactile vibration: Dynamics of sensory intensity. Journal of Experimental Psychology, 1959, 57, 210-218.

STEVENS, S. S. Tactile vibration: Change of exponent with frequency Perception \& Psychophysics, 1968, 3, 223-228.

VERRILLO, R. T. Effect of contactor area on the vibrotactile threshold. Journal of the Acoustical Society of America, 1963, 35, 1962-1966.

VERRILLO, R. T. Temporal summation in vibrotactile sensitivity. Journal of the Acoustical Society of America, 1965, 37, 843-846.

VERRILLO, R. T. Effect of spatial parameters on the vibrotactile threshold. Journal of Experimental Psychology, 1966a, 71, 570-575.

VERRILLO, R. T. Specificity of a cutaneous receptor. Perception \& Psychophysics, 1966b, 1, 149-153.

VERRILLO, R. T. A duplex mechanism of mechanoreception. In D. R. Kenshalo (Ed.), The skin senses. Springfield: C. C. Thomas, 1968.

ZWISLOCKI, J. Theory of temporal summation. Journal of the Acoustical Society of America, 1960, 32, 1046-1060.

ZWISLOCKI, J. Analysis of some auditory characteristics. In R. D. Luce, R. R. Bush, and E. Galanter (Eds.), Handbook of mathematical psychology. Vol. 3. New York: Wiley, 1965. Pp. 1-97.

ZWISLOCKI, J. Vibrotactile information transfer. Bionics Symposium, NATO Advisory Group for Aerospace Research and Development. Brussels, Belgium, 1968. In press.

\section{NOTES}

1. The authors express their appreciation to Dr. Jozef Zwislocki for his advice and for his careful reading of the manuscript. Our thanks go also to Robert Gardinier and Bernard Klock for their assistance with equipment problems.

2. This study was supported by Grant GB-5945 from the National Science Foundation and Grant NINDB-NB-03950-07 from the Nationft Institutes of Health, U.S. Department of Health, Education, and Welfate.

3. Address: Laboratory of Sensory Communication, Syracuse University, Syracuse, New York 13210.

(Accepted for publication April 16, 1969.) 